\title{
Escherichia coli verotoxigênica: isolamento e prevalência em 60 propriedades de bovinos de leite da região de Pelotas, RS, Brasil
}

\author{
Verotoxin-producing Escherichia coli: isolation and prevalence in 60 dairy cattle farms from Pelotas-RS, \\ Brazil
}

\author{
Cecília Nunes Moreira Sandrini ${ }^{\mathrm{I}}$ Murilo Anderson Pereira' ${ }^{\mathrm{II}}$ Claudiomar Soares Brod ${ }^{\mathrm{III}}$

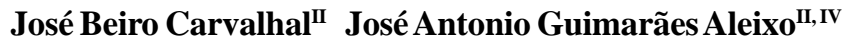

\section{RESUMO}

A produção de verotoxinas foi investigada em 1.127 isolamentos de Escherichia coli feitos a partir de 243 bovinos de leite, de água de consumo humano e animal e de amostras de leite de 60 propriedades da bacia leiteira de Pelotas, no período de dezembro de 1999 a dezembro de 2000, com o objetivo de determinar a prevalência de $\boldsymbol{E}$. coli verotoxigênicas (VTEC) nas propriedades e no rebanho, de detectar a presença de sorotipos ligados a infecções humanas e de identificar, nas propriedades e na região de Pelotas, potenciais fatores de risco de infecção para os animais. A detecção das toxinas em sobrenadante de culturas de $\boldsymbol{E}$. coli isoladas foi realizada através do ensaio de citotoxicidade em células Vero. VTEC foi isolada em 95\% (57/60) das propriedades estudadas, em 49\% (119/243) dos animais testados, em 5\% (3/60) das amostras de água de consumo humano, em 8,35\% (5/60) das amostras de água de consumo animal e em 5\% (3/ 60) das amostras de leite. A prevalência de bovinos infectados em cada propriedade variou de 0 a 100\%. Em 2,9\% (7/243) dos animais testados, foram isoladas VTEC pertencentes aos sorogrupos O157, O91 e O112, que incluem cepas responsáveis por casos de colite hemorrágica e síndrome urêmica hemolítica em humanos. Fatores de risco de contaminação, como a precipitação pluviométrica, a temperatura, o tamanho da propriedade e a concentração de animais, apresentaram evidências de influenciarem a prevalência de VTEC nos animais. Estes resultados sugerem que o grupo VTEC está amplamente distribuído na bacia leiteira de Pelotas e inclui organismos pertencentes a sorogrupos patogenicos para humanos.

Palavras-chave: Escherichia coli, verotoxinas, VTEC, bovinos.

\begin{abstract}
The production of verotoxin was investigated in 1127 Escherichia coli isolated from 243 dairy cattle, water for human and animal consumption, and milk samples from 60 dairy farms from Pelotas-Brazil, from December of 1999 to December of 2000, to determine the prevalence of verotoxigenic E. coli (VTEC) in farms, to detect the presence of serotypes involved in human infections and to identify potential risk factors for animal infection. Vero cell assay was used to detect toxins in culture supernatants from E. coli isolated. VTEC was isolated in 95\% (57/60) from farms and in 49\% (119/243) from cattle, $5 \%(3 / 60)$ from water of human consumption, in $8.35 \%(5 /$ 60) from water animal consumption and 5\% (3/60) from milk samples. The prevalence of cattle infected for each farm ranged from 0 to $100 \%$. VTEC belonging to serogroups O157, O91 and 0112 , which include strains responsible for cases of hemorrhagic colitis and hemolytic uremic syndrome in humans, were isolated from 7 (2.9\%) out of 243 cattle. Risk factors for contamination, such as amount of rain, farm size and cattle number, influenced cattle prevalence rate. These results suggest that VTEC is widely distributed among dairy cattle in the region surveyed and includes organisms from serogroups pathogenic for humans.
\end{abstract}

Key words: Escherichia coli, verotoxins, VTEC, cattle.

\section{INTRODUÇÃO}

O grupo de Escherichia coli verotoxigênicas (VTEC) é constituído por cepas que

\footnotetext{
${ }^{I}$ Curso de Medicina Veterinária, Campus de Jataí, Universidade Federal de Goiás (UFG), Jataí, GO, Brasil. E-mail: cissanm@yahoo.com.br.

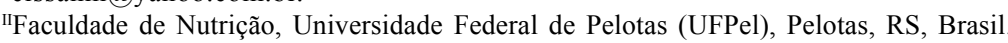

IIICentro de Controle de Zoonoses, UFPel, Pelotas, RS, Brasil. E-mail: claudiomarbrod@yahoo.com.br.

${ }^{\text {IV }}$ Centro de Biotecnologia, UFPel, CP 354, 96010-900, Pelotas, RS, Brasil. E-mail: biotjaga@ufpel.tche.br. Autor para correspondência.
} 
produzem verotoxinas, que são proteínas codificadas em fagos temperados e assim denominadas porque produzem um efeito citotóxico em células Vero (KONOWALCHUCK, 1977). As VTECs podem produzir dois tipos de verotoxinas (VT1 e VT2), que são também conhecidas como "Shiga-like toxins" (STx1 e STx2) por serem relacionadas biológica e estruturalmente com a toxina Shiga, sintetizada por Shigella dysenteriae tipo 1 (O’BRIEN et al., 1982). Estão incluídas no grupo VTEC as $\boldsymbol{E}$. coli enterohemorrágicas (EHEC), cuja cepa protótipo é o sorotipo O157:H7, associadas a doenças humanas de gravidade variável, desde os quadros diarréicos simples até casos complicados de colite hemorrágica (HC), síndrome urêmica hemolítica (HUS) e púrpura trombótica trombocitopênica (KAPER \& O'BRIEN, 1998).

A espécie bovina constitui-se no reservatório mais importante de VTECs e a maioria dos surtos de infecções humanas causadas por estas bactérias deve-se ao consumo de carne bovina mal cozida, de leite de vaca não pasteurizado e de águas de abastecimento e recreação contaminadas pelo conteúdo intestinal de animais. Diversos autores em diferentes países têm isolado sorotipos patogênicos de VTEC a partir de fezes de bovinos saudáveis (BLANCO et al., 2003; JENKINS et al., 2002; RIVERO et al., 2004; CONODERA et al., 2004). Fatores de risco envolvidos na ocorrência e distribuição de VTEC nos animais e humanos, tais como fatores de manejo na associação de excreção de VTEC patogênicas para humanos nas fezes de bovinos, vêm sendo amplamente estudados (RUGBJERG et al., 2003).

Na América do Sul, o Uruguai, o Chile e, especialmente, a Argentina apresentam incidências de infecções humanas por VTEC e de casos de HUS relativamente altas, quando comparadas às dos países do hemisfério norte, fato que é atribuído aos hábitos alimentares e à alta prevalência de VTEC em bovinos e em carne bovina (KAPER \& O'BRIEN, 1998). A Argentina apresenta o maior índice de HUS do mundo, 12,2/100.000 habitantes, sendo a principal causa de falência renal aguda e a segunda causa de falência renal crônica, e a maioria dos casos está associada ao sorotipo O157:H7 (RIVERO et al., 2004). A E. coli O157:H7 é o sorotipo predominante de VTEC nos Estados Unidos, no Canadá, no Reino Unido e no Japão, ao contrário do que ocorre na Europa Continental, na Austrália e na América do Sul, onde outros sorotipos estão mais freqüentemente envolvidos nos casos de infecções humanas (KAPER \& O’BRIEN, 1998).

A Região Sul do Brasil, embora tenha grande similaridade com os demais países do Cone Sul no que se refere ao clima, ao meio ambiente, ao sistema de exploração pecuária e aos hábitos alimentares da população, apresenta apenas comunicações pessoais de casos esporádicos de HUS e nenhum registro de isolamento de VTEC de casos de HC em humanos. Este trabalho teve por objetivo fazer um estudo de prevalência de VTEC em propriedades da bacia leiteira de Pelotas, detectar a presença de sorotipos ligados a infecções humanas e identificar, nas propriedades e nas diversas regiões que compõem a bacia, prováveis fatores de risco de infecção dos animais.

\section{MATERIAL E MÉTODOS}

Delineamento experimental

O número de propriedades a serem amostradas foi calculado utilizando-se o Programa EpiInfo 6.04 (DEAM et al., 1994), com base na freqüência porcentual esperada de propriedades com bovinos reservatórios de VTEC em países próximos ao Brasil, já que não havia informações sobre este país. Usando uma expectativa de prevalência de $80 \%$, com margem de erro de $10 \%$ e nível de confiança de $95 \%$, obteve-se uma amostra de 60 propriedades. O critério de inclusão das propriedades foi o de seu proprietário ser produtor cooperado da Cooperativa Sul-Riograndense de Laticínios (COSULATI) do Município de Pelotas. Estas foram escolhidas a partir de uma listagem de todos os produtores da Cooperativa, sendo classificados por extrato de produção média de leite por dia.

Para assegurar que os diferentes tipos de propriedades existentes na bacia leiteira estivessem representados no estudo, elas foram divididas em três estratos de produção: 30 produtoras de até 30 litros/ leite/dia, 12 produtoras entre 30 e 50 litros/leite/dia; e 18 produtoras de mais de 50 litros/leite/dia. A amostra aleatória sistemática foi extraída calculando-se, em cada um dos extratos, um pulo pela razão entre o número total de produtores associados à Cooperativa e o número de propriedades a serem amostradas. As propriedades ficaram distribuídas em quatro regiões, cada uma contendo um grupo de 15 propriedades, que foi visitado em uma única estação do ano, sendo as regiões numeradas de 1 a 4 (Figura 1). Cada região conteve um número proporcional de propriedades dos três estratos. Foi utilizado um questionário estruturado, aplicado ao produtor para identificação de fatores de risco de infecção para os animais e de contaminação das propriedades. Esse questionário era composto de três partes: uma sobre as características físicas, biológicas e econômico-sociais da propriedade, outra sobre a quantidade, disponibilidade e condições sanitárias da água de abastecimento humano e animal e a terceira sobre cada animal (características de 
hospedeiro) incluído na amostragem, totalizando 78 questões.

O estudo foi realizado no período de um ano, de dezembro de 1999 a dezembro de 2000, sendo levantadas prevalências e investigados prováveis fatores de risco. Nas 60 propriedades estudadas, que continham um total de 1.136 bovinos distribuídos em 1.216 hectares, foram coletadas amostras de fezes de 243 animais, distribuídos em três grupos etários: 50\% de terneiros de até 11 meses de idade e o restante dividido entre novilhas e vacas adultas. A coleta foi feita em 4 animais de cada propriedade, uma única vez, escolhidos a partir de uma amostragem aleatória simples, no momento da visita, sorteando-se dois terneiros, uma novilha e uma vaca adulta. Coletaram-se também pelo menos uma amostra de cada fonte de abastecimento de água de consumo humano e animal da propriedade e de leite cru.

Coleta e processamento das amostras

Amostras de fezes foram coletadas através de "swab" retais, colocadas em meio de transporte (Stuart) e levadas ao laboratório em caixas isotérmicas para processamento. As amostras de água e leite cru foram coletadas em volumes de $100 \mathrm{~mL}$ em frascos estéreis. As amostras de fezes foram semeadas diretamente em ágar EMB-Levine e incubadas a $37^{\circ} \mathrm{C}$, por 24 horas. As amostras de água e leite, após passarem por enriquecimento em caldo lauril sulfato triptose por $24 \mathrm{~h} \mathrm{a} 37^{\circ} \mathrm{C}$, também foram semeadas em ágar EMB-Levine. De cada amostra, foram escolhidas 5 colônias suspeitas de serem $\boldsymbol{E}$. coli para confirmação através dos testes IMViC (indol, vermelho de metila, Voges Proskauer e utilização de citrato), motilidade e produção de H S em meio SIM, fermentação da lactose e sorbitol. Cađa isolamento de $\boldsymbol{E}$. coli foi estocado a temperatura ambiente em $0,75 \%$ de ágar nutriente, para realização posterior dos testes de citotoxicidade e sorotipagem.

Produção e detecção de verotoxinas em Células Vero A produção de verotoxinas foi determinada pela prova de citotoxicidade em células Vero, como descrito por BLANCO et al. (1996a), com modificações. Os isolamentos estocados foram inoculadas em $1 \mathrm{~mL}$ de TSB (caldo de triptona de soja) e submetidas a crescimento por 18 horas a $37^{\circ} \mathrm{C}$, em agitador orbital a 240rpm. Após isso, o cultivo foi centrifugado (18.000 g) por 25 minutos, a $4^{\circ} \mathrm{C}$. Os sobrenadantes contendo as toxinas foram estocados a $-18^{\circ} \mathrm{C}$ até o momento do teste. Para o ensaio de citotoxicidade, inicialmente, prepararam-se placas de cultivo celular de 96 cavidades que contivessem uma monocamada de células Vero confluentes. Para isso, foram cultivadas, em cada cavidade, $10^{5}$ células suspensas em $100 \mu \mathrm{L}$ de Meio Essencial Mínimo (MEM) (Cultilab, Campinas) suplementado com $10 \%$ de soro fetal bovino (Cultilab), $1 \%$ de L-Glutamina (Cultilab), 100.000 UI de penicilina $\mathrm{G}, 100 \mathrm{mg}$ de estreptomicina e $25 \mu \mathrm{g}$ de anfotericina B por litro, por 24 horas, a $37^{\circ} \mathrm{C}$ e atmosfera de $5 \%$ de $\mathrm{CO}_{2}$.

A seguir, prepararam-se três diluições (1/4, $1 / 8,1 / 16)$ das preparações de toxinas e incubaram-se as placas a $37^{\circ} \mathrm{C}$ e $5 \%$ de $\mathrm{CO}_{2}$. As alterações morfológicas nas células foram observadas após $24 \mathrm{e}$ 48 horas de incubação com um microscópio de contraste de fase invertido. Uma cepa de $\boldsymbol{E}$. coli $\mathrm{K}-12$ foi utilizada como controle negativo e uma cepa de $\boldsymbol{E}$. coli 0157:H7 (cedida por T. Yano, UNICAMP) foi utilizada como controle positivo em cada placa.

\section{Sorotipagem}

A sorotipificação das cepas de $\boldsymbol{E}$. coli produtoras de verotoxinas foi realizada no Laboratório de Enterobactérias da FIOCRUZ-RJ. Na sorotipificação, foram utilizados apenas soros anti-E. coli patogênicas para humanos (EPEC grupo A: O26, O55, O111, O119, O127; EPEC grupo B: O18, O25, O86, O91, O128, O158; EPEC grupo C: O44, O112A, O112B, O125, O126, O142; EIEC grupo 1: O29, O112AC, O125, 0126, 0142; EIEC grupo 2: O28, O114, O124, O136, O143, O152, O157).

\section{Análises estatísticas}

Os dados foram computados no programa Epi Info Versão 6.04 (DEAM et al., 1994). Foi usado o $\chi^{2}$ de Mantel-Haenzsel para tendência linear e "razão de chances" (OR), no intervalo de confiança de 95\%, para avaliar os efeitos de diferentes grupos etários, prováveis fatores de risco ambientais (NIELSEN et al., 2002; RUGBJERG et al., 2003) e manejo na prevalência de animais infectados com VTEC.

\section{RESULTADOS E DISCUSSÃO}

Prevalência de VTEC em bovinos de leite sadios Foi investigada a produção de verotoxinas em 1.127 isolamentos de Escherichia coli feitos a partir de 243 bovinos aparentemente sadios das 60 propriedades leiteiras estudadas. Encontrou-se VTEC em 48,9\% (119/243) dos animais e em 95\% (57/60) das propriedades. Quanto aos grupos etários, foram obtidos isolamentos de VTEC em 43,5\% (53/122) dos terneiros, $56,8 \%(33 / 58)$ das novilhas e 52,4\% (33/63) das vacas adultas. Do total de isolamentos, $29,1 \%$ (328/1127) foram produtores de verotoxinas. A proporção de animais infectados em cada propriedade variou de 0 a $100 \%$. Em 5\% das propriedades (3/60), não foi obtido nenhum 
isolamento de VTEC. Em 23,3\% das propriedades (14/ 60 , foram obtidos isolamentos de VTEC em $25 \%$ dos animais. Em 33,4\% das propriedades (20/60), foram obtidos isolamentos de VTEC em $50 \%$ dos animais. Em $25 \%(15 / 60)$ e $13,3 \%(8 / 60)$ das propriedades, respectivamente, foram obtidos isolamentos de VTEC em $75 \%$ e $100 \%$ dos animais.

Fatores de risco para animais reservatórios de VTEC $\mathrm{Na}$ análise dos resultados referentes aos prováveis fatores de risco ambientais de infecção, a temperatura média mensal acima de $22,5^{\circ} \mathrm{C}$ $(1,74<\mathrm{OR}<12,02 ; \mathrm{P}=0,003)$ e o índice pluviométrico inferior a $70 \mathrm{~mm}$ ou superior a $140 \mathrm{~mm}(1,43<\mathrm{OR}<4,66$; $\mathrm{P}<0,001)$ sugeriram, pelos resultados encontrados, influências significativas na infecção dos animais por VTEC. Observou-se, também, que as propriedades pequenas $(<20$ hectares) apresentaram um número maior de animais infectados por $\operatorname{VTEC~}(1,20<\mathrm{OR}<3,71$; $\mathrm{P}=0,004)$ do que as propriedades maiores $(>20$ hectares $)$. Em propriedades onde as vacas permaneciam na cocheira durante a noite, a prevalência de animais infectados com VTEC foi maior do que em propriedades que deixavam as vacas soltas no campo ou em piquetes $(1,01<\mathrm{OR}<3,09 ; \mathrm{P}=0,003)$. O mesmo fato ocorreu em propriedades com número total de bovinos inferior a $14(1,25<\mathrm{OR}<3,87 ; \mathrm{P}=0,003)$ (Tabela 1). A distribuição espacial da prevalência de animais com VTEC está expressa na figura 1 e na tabela 2 .

Fatores de proteção

Em propriedades (Tabela 1) onde era realizado algum tipo de tratamento da água, a prevalência de animais com VTEC foi menor do que nas propriedades que não realizavam qualquer tipo de tratamento $(0,25<\mathrm{OR}<0,90 ; \mathrm{P}=0,013)$. Com relação à idade, terneiros de até 11 meses de idade apresentaramse menos infectados que as novilhas e vacas adultas $(0,32<\mathrm{OR}<0,98 ; \mathrm{P}=0,02)$.

Prevalência de VTEC em amostras de água e leite

Foi analisada uma amostra de água de consumo humano, uma de água de consumo animal e uma de leite cru, correspondente a cada uma das 60 propriedades estudadas. A prevalência de VTEC nestas amostras foi de 5\% (3/60) nas amostras de água de consumo humano, de $8,35 \%(5 / 60)$ nas amostras de água de consumo animal e de 5\% (3/60) nas amostras de leite.

Sorotipagem

Entre os 328 isolamentos de VTEC, 2,4\% (8/ 328) foram classificados como sorotipos patogênicos para humanos e o restante não pôde ser tipificado com a bateria de soros utilizada. As cepas tipificadas pertenciam aos sorotipos O157:H?, O157:NM, O91:NM e O112:NM. Estas cepas foram isoladas em 2,9\% (7/ $243)$ dos animais, em 11,7\% (7/60) das propriedades. Com relação à idade dos animais infectados com os sorotipos potencialmente patogênicos, $71,4 \%(5 / 7)$ tinham idade inferior a 12 meses e 28,6\% (2/7) tinham idade superior a 84 meses. Quanto à raça dos animais infectados, $71,4 \%(5 / 7)$ das cepas foram isoladas de animais da raça Jersey (Tabela 3 ).

As prevalências de VTEC encontradas neste estudo, de 43,5\% (53/122) em terneiros de até 11 meses de idade, de 56,8\% (33/58) em novilhas e de $52,4 \%(33 / 63)$ em vacas adultas, foram superiores às encontradas em países como Espanha, França, Austrália, Japão, Alemanha, Itália, Inglaterra, Egito e

Tabela 1 - Fatores de risco ou proteção para infecção/contaminação por $\boldsymbol{E}$. coli verotoxigênica (VTEC), na bacia leiteira do município de Pelotas, RS, no período de dezembro de 1999 a dezembro de 2000.

\begin{tabular}{|c|c|c|c|c|c|}
\hline \multirow{2}{*}{ Fator de risco ou proteção** } & \multicolumn{2}{|c|}{ VTEC (\%) } & \multirow[t]{2}{*}{ OR } & \multirow[t]{2}{*}{ CI $95 \%$} & \multirow[t]{2}{*}{$\mathrm{P}$} \\
\hline & pos. & neg. & & & \\
\hline Temperatura acima de $22,5^{\circ} \mathrm{C}^{*}$ & 22,7 & 6,1 & 4,28 & $1,74-12,02$ & 0,0003 \\
\hline Precipitação pluviométrica inferior a $70 \mathrm{~mm}$ e superior a $140 \mathrm{~mm} *$ & 74,8 & 53,5 & 2,58 & $1,43-4,66$ & 0,0006 \\
\hline Propriedades com menos de 14 bovinos* & 57,1 & 37,7 & 2,20 & $1,25-3,87$ & 0,0030 \\
\hline Propriedades com menos de 20 hectares* & 58,8 & 40,4 & 2,11 & $1,20-3,71$ & 0,0048 \\
\hline Vacas permanecem à noite em cocheira* & 62,2 & 48,2 & 1,76 & $1,01-3,09$ & 0,0032 \\
\hline Idade do animal inferior a 12 meses** & 44,5 & 58,8 & 0,56 & $0,32-0,98$ & 0,0297 \\
\hline Propriedades com tratamento de água** & 66,4 & 80,7 & 0,47 & $0,25-0,90$ & 0,013 \\
\hline
\end{tabular}

OR - "Razão de Chances".

CI - Intervalo de Confiança.

VTEC - E. coli verotoxigênica.

Ciência Rural, v.37, n.1, jan-fev, 2007. 


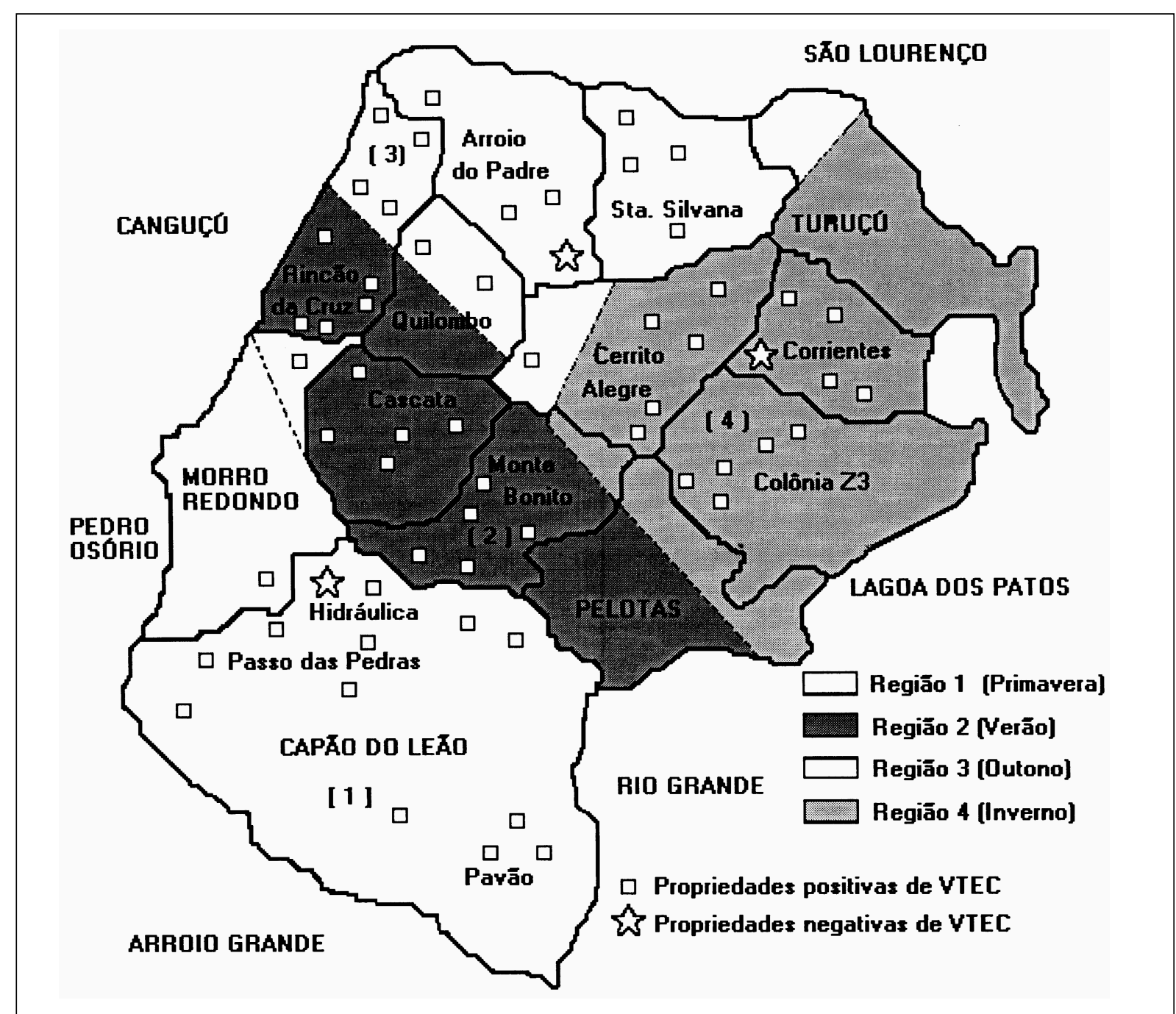

Figura 1 - Mapa com distribuição espacial das 60 propriedades amostradas no período de dezembro de 1999 a dezembro de 2000 , para determinação de $\boldsymbol{E}$. coli verotoxigênica (VTEC) em bovinos da bacia leiteira do município de Pelotas, RS.

Escócia (BLANCO et al., 1996b; GALLIEN et al., 1997; HEUVELINK et al., 1998; COBBOLD \& DESMARCHELIER, 2000; PRADEL et al., 2000; JENKINS et al., 2002; PAIBA et al., 2003). Na Argentina, foi registrada uma prevalência de VTEC de 44\% em vacas sadias prontas para abate (SANZ et al., 1998), o que se aproxima dos valores encontrados neste trabalho.

$\mathrm{Na}$ análise dos prováveis fatores de risco, observou-se que a variável "tamanho da propriedade" influenciou a prevalência de VTEC, uma vez que propriedades com menos de 20 hectares e com número total de bovinos inferior a 14 apresentaram maior prevalência de VTEC do que as outras propriedades maiores e com um maior número de animais. Isto pode ser parcialmente explicado pelo fato de que os pequenos produtores usavam menos tecnologia, dispunham de uma infra-estrutura física inadequada e eram menos esclarecidos quanto às condições de higiene e manejo de animais estabulados. Confirmando esta explicação, foi observado que a prevalência de animais com VTEC foi maior nas propriedades em que as vacas permaneciam na cocheira durante a noite do que nas propriedades que deixavam os animais soltos no campo, considerando-se, nestes casos, o fator aglomeração dos animais, que favorece a transmissão do agente.

Em relação à idade, observou-se um fator de proteção para terneiros de até 12 meses de idade, o que contrasta com o normalmente registrado, que é de animais jovens apresentarem maiores prevalências de VTEC. Embora a prevalência de VTEC neste grupo etário tenha sido menor que a esperada, ele conteve a 
Tabela 2- Prevalência de animais contaminados por E. coli verotoxigênica (VTEC) em cada região da bacia leiteira do município de Pelotas, RS.

\begin{tabular}{|c|c|c|c|c|c|c|}
\hline \multirow{2}{*}{ Região } & \multirow{2}{*}{ Temperatura média ${ }^{\circ} \mathrm{C}^{*}$} & \multirow{2}{*}{ Precipitação média mm** } & \multicolumn{2}{|c|}{ Animais } & \multirow{2}{*}{ Total } & \multirow{2}{*}{ Freqüência \% } \\
\hline & & & VTEC & Não VTEC & & \\
\hline 1 & 15,90 & 96,85 & 20 & 42 & 62 & 32,3 \\
\hline 2 & 22,03 & 79,60 & 40 & 21 & 61 & 65,6 \\
\hline 3 & 15,10 & 159,93 & 28 & 32 & 60 & 46,7 \\
\hline 4 & 12,35 & 157,90 & 31 & 29 & 60 & 51,7 \\
\hline Total & - & - & 119 & 124 & 243 & 48,9 \\
\hline
\end{tabular}

*Temperatura média de cada estação e região.

**Precipitação pluviométrica média de cada estação e região (Boletim Agroclimatológico. Estação Agroclimatológica de Pelotas. Convênio Embrapa/UFPel/INMET).

maioria dos isolamentos pertencentes a sorogrupos patogênicos para humanos, confirmando o que foi observado em outros trabalhos (COBBOLD \& DESMARCHELIER, 2000; HEUVELINK et al., 1998; NIELSEN et al., 2002; ORDEN et al., 2002; PAIBA et al., 2003).

A ocorrência de altas temperaturas aparentemente influiu positivamente no número de animais infectados (Tabela 2), o que, embora com freqüência muito superior, também foi observado em outros trabalhos (KAPER \& O'BRIEN, 1998; BONARDI et al., 1999). Temperaturas elevadas favorecem a sobrevivência e multiplicação de $\boldsymbol{E}$. coli no ambiente, possibilitando sua disseminação entre os animais.

Com relação à precipitação pluviométrica mensal (Tabela 1), a prevalência de animais com VTEC foi maior em meses com precipitação pluviométrica dentro do intervalo inferior a $70 \mathrm{~mm}$ e superior a $140 \mathrm{~mm}$. Isto poderia ser explicado pelo fato de, em períodos de escassez de chuva, ocorrer maior aglomeração de animais ao redor das fontes de abastecimento, o que facilita a transmissão interanimal do agente patogênico.

Tabela 3 - Sorotipos de $\boldsymbol{E}$. coli verotoxigênica (VTEC) isolados de bovinos sadios na bacia leiteira do município de Pelotas-RS.

\begin{tabular}{lcl}
\hline Sorotipos & Número de isolamentos & $\begin{array}{c}\text { Número de animais } \\
(\mathrm{T} / \mathrm{N} / \mathrm{V})^{*}\end{array}$ \\
\hline O157:H? & 2 & $(1 / 0 / 1)$ \\
O157:NM & 1 & $(1 / 0 / 0)$ \\
O91:NM & 3 & $(2 / 0 / 1)$ \\
O112:NM & 1 & $(1 / 0 / 0)$ \\
Não & 320 & $(48 / 33 / 33)$ \\
sorotipáveis & & \\
\hline
\end{tabular}

*terneiros/novilhas/vacas.
Já em períodos de excesso de chuva, ocorre a dispersão superficial do agente por carreamento, propiciando sua transmissão.

No presente trabalho, foi detectado VTEC em 48,9\% (119/243) dos animais e em 95,0\% (57/60) das propriedades examinadas. Já os sorogrupos de VTEC patogênicas (O157, O91 e O112) foram encontrados em apenas $2,9 \%$ dos animais e em $11,7 \%$ das propriedades. Estudos de prevalência de sorotipos patogênicos em bovinos têm sido realizados em várias partes do mundo. Nos Estados Unidos, o sorotipo predominante é o O157:H7, que foi encontrado em $63 \%$ dos confinamentos investigados em 13 estados americanos (HANCOCK et al., 1997) e que é encontrado em 0,5 a $2 \%$ de amostras de fezes dos animais (HANCOCK et al., 1998). Na Europa continental, observa-se a ocorrência de diversos sorogrupos patogênicos de VTEC. Estudos realizados em vários países isolaram de fezes bovinas VTEC dos sorogrupos O26, O111, $\mathrm{O} 103$ e $\mathrm{O} 128$ em proporções variando de 4,2 a 29\%. O sorogrupo $\mathrm{O} 157$ foi isolado em 1 a 3,6\% dos animais (CAPRIOLI \& TOZZI, 1998; NIELSEN et al., 2002; ORDEN et al., 2002). Na Argentina, onde há a maior incidência mundial de HUS, há poucos estudos sobre a ocorrência de sorogrupos de VTEC patogênicas em bovinos. Em um trabalho desenvolvido por ORSKOV et al. (1987), foi verificada a prevalência de $\boldsymbol{E}$. coli O157:H7 em 7,7\% das amostras fecais de terneiros, ao passo que, em outro trabalho, SANZ et al. (1998) isolaram VTEC em 44\% das vacas prontas para o abate, sem, entretanto, detectar nenhuma O157:H7.

\section{CONCLUSÃO}

Foi comprovada a presença de Escherichia coli verotoxigênica em amostras de fezes de bovinos de leite, em amostras de água ambiental, de água de

Ciência Rural, v.37, n.1, jan-fev, 2007. 
consumo humano e em amostras de leite cru, em propriedades rurais da bacia leiteira de Pelotas-RS. A prevalência de VTEC na bacia leiteira de Pelotas foi de $49 \%(119 / 243)$ dos animais testados, pertencentes a 95\% (57/60) das propriedades estudadas, onde a prevalência de bovinos infectados variou de 0 a $100 \%$. Esta prevalência é similar à encontrada em outros países. Alguns isolamentos de VTEC encontrados pertenceram a sorogrupos potencialmente patogênicos para humanos (O157, O91 e O112).

Com relação aos fatores de risco, condições ambientais, como escassez ou excesso de chuva, e elevadas temperaturas contribuíram significativamente para o aumento da prevalência de animais infectados com VTEC. Características da propriedade, tais como tamanho (pequenas propriedades), número de animais (pequeno número de animais) e encerramento de vacas em cocheiras à noite, foram relacionadas à maior prevalência de animais infectados com VTEC. Embora o maior número de isolamentos de VTEC não tenha ocorrido nos animais jovens, a este grupo etário pertenceu a maioria dos isolamentos de sorogrupos patogênicos para humanos.

\section{AGRADECIMENTOS}

À Coordenação de Aperfeiçoamento de Pessoal de Nível Superior (CAPES), pela bolsa para CNMS e à Fundação de Amparo à Pesquisa do Estado do Rio Grande do Sul (FAPERGS), pelo Auxílio No 99/0985.7.

\section{REFERÊNCIAS}

BLANCO, M. et al. Distribuition and characterization of faecal verotoxin-producing Escherichia coli (VTEC) isolated from healthy cattle. Veterinary Microbiology, Amsterdam, Holanda, v.54, p.309-319, 1996a.

BLANCO, M. et al. Prevalence and characteristics of human and bovine verotoxigenic Escherichia coli strains isolated in Galicia (north-western Spain). European Journal Epidemiology, Holanda, v.12, p.13-19, 1996 b.

BLANCO, J. et al. Verotoxin-producing Escherichia coli in Spain: prevalence, serotypes, and virulence genes of 0157:H7 and non-O157 VTEC in ruminants, raw beef products, and humans. Experimental Biology and Medicine, Maywood, New Jersey, USA, v.228, n.4, p.345-351, 2003.

BONARDI, S. et al. Isolation of Verocytotoxin-producing Escherichia coli $0157: \mathrm{H} 7$ from cattle at slaughter in Italy Veterinary Microbiology, Amsterdam, Holanda, v.67, p.203$211,1999$.

CAPRIOLI, A.; TOZZI, A.E. Epidemiology of toxin-producing Escherichia coli infections in Continental Europe. In: KAPER, J.B.; O'BRIEN, A.D. Escherichia coli O157:H7 and other Shiga Toxin-Producing E. coli strains. Washington: ASM, 1998. Cap.5, p.38-48.

COBBOLD, R.; DESMARCHELIER, P. A longitudinal study of
Shiga-toxigenic Escherichia coli (STEC) prevalence in three Australian dairy herds. Veterinary Microbiology, Amsterdam, Holanda, v.71, p.125-137, 2000.

CONODERA, G. et al. Verocytotoxin-producing Escherichia coli $\mathrm{O} 157$ in minced beef and dairy products in Italy. International Journal of Food Microbiology, Amsterdam, Holanda v.96, n.1, p.67-73, 2004.

DEAM, A.G. et al. Epi Info Version 6.0. A word processing, database, and statistics program for epidemiology on microcomputers. Atlanta, GA.: Centers for Disease Control and Prevention, 1994. 278p

GALLIEN, P. et al. Detection of STEC and epidemiological investigations in surrounding of a HUS patient. Berliner Und Munchener Tierarztliche Wochenschrift, Berlim, v.110, p.342-346, 1997.

HANCOCK, D.D. et al. Epidemiology of Escherichia coli O157 in feedlot cattle. Epidemiology Infection. Washington, USA, v.60, p.462-465, 1997.

HANCOCK, D.D. et al. Ecology of Escherichia coli O157:H7 in cattle and impact of management practices. In: KAPER, J.B.; O'BRIEN, A.D. Escherichia coli O157:H7 and other Shiga Toxin-Producing E. coli Strains. Washington: ASM, 1998. p.85-91.

HEUVELINK, A.E. et al. Isolation and characterization of Verocytotoxin-producing Escherichia coli O157 strains from dutch cattle and sheep. Journal Clinical of Microbiology, Washington, USA, v.36, p.878-882, 1998.

JENKINS, C. et al. An eight-month study of a population of verocytotoxigenic Escherichia coli (VTEC) in a Scottish cattle herd. Journal of Applied Microbiology, Oxford, Inglaterra, v.93, n.6, p.944-953, 2002.

KAPER, J.B.; O'BRIEN, A.D. Escherichia coli 0157:H7 and Other Shiga Toxin-Producing E. coli Strains. Washington, USA: ASM, 1998. 459p.

KONOWALCHUK, J. et al. Vero response to a cytotoxin of Escherichia coli. Infection and Immunity, Washington, v.18, p.775-779, 1977.

NIELSEN, E.M. et al. Influence of age, sex and herd characteristics on the occurrence of verocytotoxin-producing Escherichia coli O157 in Danish dairy farms. Veterinary Microbiology, Amsterdam, Holanda, v.88, n.3, p.245-257, 2002.

O'BRIEN A.D. et al. Production of Shigella dysenteriae type 1-like cytotoxin by Escherichia coli. Journal Infection Disease, Chicago, Estados Unidos, v.146, p.763-769, 1982.

ORDEN, J.A. et al. Verotoxin-producing Escherichia coli (VTEC), enteropathogenic E. coli (EPEC) and necrotoxigenic $\boldsymbol{E}$. coli (NTEC) isolated from healthy cattle in Spain. Journal of Applied Microbiology, Oxford, v.93, n.1, p.29-35,2002.

ORSKOV, F. et al. Cattle as reservoir of verotoxin-producing Escherichia coli 0157:H7. Lancet, Connectcut, v.1, n.2, p.276, 1987.

PAIBA, G.A. et al. Prevalence of faecal excretion of verocytotoxigenic Escherichia coli O157 in cattle in England and Wales. Veterinary Record, London, v.153, n.12, p.347353,2003 
PRADEL, N. et al. Prevalence and characterization of Shiga Toxin-Producing Escherichia coli isolated from cattle, food, and children during a one-year prospective study in France. Journal Clinical of Microbiology, Washington, v.38, p.1023-1031, 2000.

RIVERO, M.A. et al. Enterohemorrhagic Escherichia coli and hemolytic-uremic syndrome in Argentina. Medicina, Buenos Aires, v.64, n.4, p.352-356, 2004.
RUGBJERG, H. et al. Risk factors associated with faecal shedding of verocytotoxin-producing Escherichia coli O157 in eight known-infected Danish dairy herds. Preventive Veterinary Medicine, Amsterdam, v.58, n.3/4, p.101-113, 2003.

SANZ, M.E. et al. Prevalence of bovine verotoxin-producing Escherichia coli in Argentina. European Journal Epidemiology, Holanda, v.14, n.4, p.399-403, 1998. 